\title{
LDL Cholesterol Testing Device using Serial Reflected Face-to-Face Mirror System
}

\author{
Min-Seong Choi and Jae-Chem Yoo* \\ School of Information and Communication Engineering, Sungkyunkwan University, Suwon 440-746, Korea
}

(Received May 15, 2013 : revised July 29, 2013 : accepted July 29, 2013)

\begin{abstract}
A low density lipoprotein (LDL) cholesterol testing device, structured with serial reflected face-to-face mirror (SRM) allowing spectrophotometry measurements, is presented. The spectrophotometry has been employed to measure the amount of light that a sample absorbs, but it generally should have had path length longer than $10 \mathrm{~mm}$ to secure enough sensitivity. Such requirement of path length has often been problematic in implementing a thin type of lab on a disc (LOD). We developed the SRM system which was implemented in a detection chamber with $1.4 \mathrm{~mm}$ thickness, providing path length longer than $10 \mathrm{~mm}$, and thus straightforwardly being applicable to LOD as thin as a compact disc. The experimental results show that the SRM system gives not only a much thinner design compared to the conventional spectrophotometry-based LOD but also a comparable performance to already commercialized spectrometers.
\end{abstract}

Keywords: Lab-on-a-disc, Serial reflection, Optical sensing device, Optical path length

OCIS codes : (040.1880) Detection; (120.5700) Reflection; (280.1415) Biological sensing and sensors; (280.4788) Optical sensing and sensors

\section{INTRODUCTION}

LDL cholesterol has been known as bad cholesterol because its high level is closely associated with an increased risk of heart attack and stroke [1]. Recently, a large number of bio-assays for screening high level LDL cholesterol have been reported in the literature since it has been recognized that those assays can greatly increase the chances for successful treatment. They can be classified into three categories according to the technology used: immunosensor utilizing surface plasmon resonance phenomenon on immobilized self-assembled monolayer (SAM) [2, 3], amperometric and potentiometric detector to measure electrochemical potential or current generated from the enzymatic reaction of cholesterol [4-8] and spectrophotometer to measure the absorbance of light by the sample at a given wavelength $[9,10]$. Among these sensors the spectrophotometer is expected to be the most easily applicable to LOD, commonly known as a compact disc (CD) based centrifugal microfluidic platform, because of its noncontact measurement. The spectroscopy technique has been most frequently employed in life science laboratories as a method to determine the concentration of a certain substance in a solution. Spectrophotometry approach to LOD allows the automation of complex fluidic tasks by centrifugal force as well as providing contact-free measurement of bio-chemical reaction. That's the reason why many biochemical analyzers have been focused on developing spectrophotometry-based LOD [11-13]. Despite such advantages, however, there are still some difficulties in obtaining LOD as thin as a compact disc because of Lambert-Beer's law that requires a long path length, typically $10 \mathrm{~mm}$ that is the distance that light travels through a sample, to offer enough sensitivity as a spectrometer. It consequently led to the development of LOD with a $10 \mathrm{~mm}$ thick detection chamber $[14,15]$. To solve the thickness issue, J. Steigert and M. Grumann [16, 17] integrated two V-grooved total internal reflections (TIR) into LOD and thereby achieved the $10 \mathrm{~mm}$ path length with no more than about $1.4 \mathrm{~mm}$ thick detection cell. However, this approach requires inevitably more designing area to be available on LOD than is traditionally occupied since it needs a detection cell and two V-grooves azimuthally arranged on the LOD.

In this paper, these problems are overcome through the use of a serial reflected face-to-face mirror (SRM) that offers longer path length and at the same time eliminates the necessity of those V-grooves.

\footnotetext{
*Corresponding author: yoojc@skku.edu

Color versions of one or more of the figures in this paper are available online.
} 


\section{MATERIALS}

The conventional CD was modified to suit our SRM system made from three polycarbonate layers by an injection molding machine (FA-DRA1F, Panasonic, Japan). Those layers were combined with double sided adhesive films (Tesa 4928, Germany, thickness: $0.125 \mathrm{~mm}$ ), consisting of a transparent PET film backing and a modified acrylic adhesive, under pressure to finally construct a detection chamber for storing biological samples to be analyzed and to form aface-to face mirror consisting of lower and upper mirrors. Polycarbonate has good properties such as high light transmittance in the visible light range (over $85 \%$ ), easy molding and low water absorption $(0.16 \sim 0.35 \%)$. The two mirrors were coated with aluminum to a thickness of about $200 \mathrm{~nm}$ and were set to face each other, causing multiple reflections between them and in turn making the effective path length of light longer. Fig. 1 shows the whole schematic diagram of the proposed SRM system designed on a compact disc with the diameter of $120 \mathrm{~mm}$ where the test sample is contained within a detection chamber with a volume of approximately $50 \mu$. The chamber has a dimension of width $6 \mathrm{~mm}$ depth $1.4 \mathrm{~mm}$ effective length $5 \mathrm{~mm}$. 99\% pure aluminum film was deposited on one side of the polycarbonate layers 1 and 3, by a vacuum decomposition process called cathode-sputtering (SWIVEL 1.8, UNAXIS, USA). Then the layers, with appropriate masks, were placed below

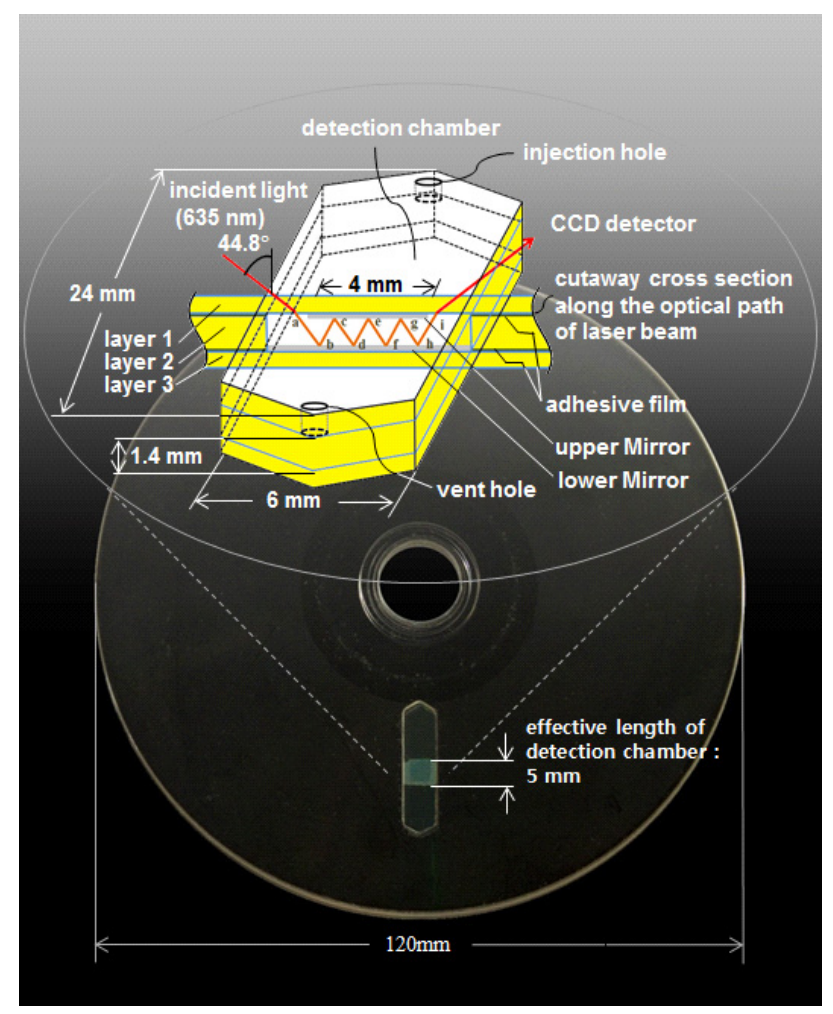

FIG. 1. SRM system implemented on a compact disc in which lower \& upper mirrors are bonded in a face-to-face configuration to provide multiple reflections. cathode and at some distance from the material being evaporated. The masks placed on the layers allow a specific pattern to be created. The layers 1 and 3 were, respectively, masked by patterns with a rectangular opening of $4 \mathrm{~mm} \times 5 \mathrm{~mm}$ and $6 \mathrm{~mm} \times 5 \mathrm{~mm}$. These opening patterns leave upper and lower mirrors on each surface of the layers after the sputtering cycle.

Our SRM system is designed to have the upper mirror slightly smaller in size compared to the lower one in order to allow the incident light to easily enter the chamber and exit from the chamber at the final reflection point without any interruption. A potential of about 3 kilovolts is applied between the anode and cathode of the sputterer. When the sputtering cycle is completed, the vacuum is released and the mask is removed from the polycarbonate layer, leaving the thin aluminum film for the mirrors on all parts of the layers exposed by the open portions of the mask.

\section{SRM (SERIAL REFLECTED FACE-TO-FACE MIRROR) SYSTEM}

In this experiment, a two dimensional-charge coupled device (2D-CCD) detector is used as a sensor of a spectrophotometer to detect the spectral intensity of LDL cholesterol. The test sample in the chamber is illuminated by laser beam (LM-6305MR, Lanics co., Korea) with wavelength of 635 $\mathrm{nm}$ and then the 2D-CCD detector (UI-1220-M, uEye co., German) with $752 \times 480$ pixels at 8 bit dynamics measures the light that passes through it. First, the incident light passes through layer 1 and starts reflecting when it reaches the surface of the lower mirror. The reflected beam keeps traveling along the face-to-face mirror formed between layers 1 and 3 making multiple reflections and finally arrives at the CCD detector via layer 1 with the absorbance of the light proportional to the concentration of the chemical species that the light passes through (See Fig. 2).

The absorbance (A) can be given according to the LambertBeer's law, $A=\log _{10}\left(I_{0} / I\right)$ where $I_{0}$ and $I$, respectively, correspond to the intensity of the light passing through the SRM chamber before and after enzymatic reaction. These intensities were calculated by removing noise of the image from the CCD detector and taking an average over all pixel values. The incidence angle to the SRM was determined under the consideration that the path length has to be longer than at least $10 \mathrm{~mm}$, giving an appropriate degree of sensitivity in spectroscopy. Theoretical calculation based on the physical configuration of the SRM shows that a longer path length than $10 \mathrm{~mm}$ can be obtained as long as the incidence angle is set to be between $31.3^{\circ}$ and $44.8^{\circ}$, and thus we finally chose the incidence degree as $44.8^{\circ}$, which gives a maximum path length. When the incidence angle was set to be $44.8^{\circ}$, there were seven serially reflected reflections, resulting in an effective path length of 11.3 $\mathrm{mm}$. 


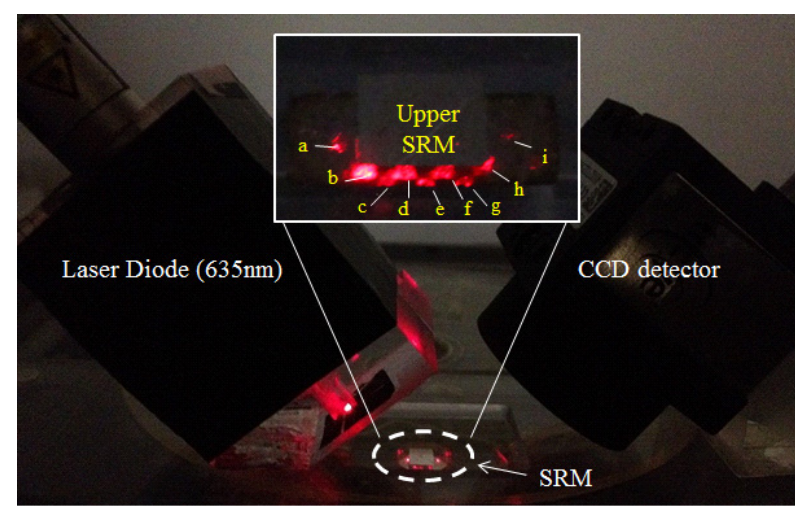

FIG. 2. Experimental setup of the SRM system where the incident ray passing through the entrance portion " $a$ " travels along the SRM with making seven reflections $(b \rightarrow c \rightarrow d \rightarrow$ $e \rightarrow f \rightarrow g \rightarrow h$ ) on lower \& upper mirrors and finally reaches CCD camera via the exit portion " $i$ ".

\section{EXPERIMENTAL RESULTS AND DISCUSSION}

In this experiment, an enzymatic reaction called COD-POD method was used to determine the concentration of LDL cholesterol dissolved in a standard cholesterol solution. This involves hydrolyzing cholesterol esters by cholesterol esterase (ChE) into LDL cholesterol, and then the LDL cholesterol is oxidased by cholesterol oxidase (COD) to produce 4-Cholestenone and an equimolar amount of hydrogen peroxide $\left(\mathrm{H}_{2} \mathrm{O}_{2}\right)$. In the presence of peroxidase (POD), the hydrogen peroxide oxidizes p-hydroxy-benzene sulfonate and 4-aminoantipyrine to give a quinoneimine dye colored in violet. The concentration of cholesterol was determined by measuring the absorbance (A) of the light at wavelength $635 \mathrm{~nm}$. To evaluate the performance of our SRM system involved in coefficient of variation $(\mathrm{CV})$ and coefficients of determination $\left(R^{2}\right)$, the amount of light absorbed by a set of standard cholesterol solutions of known concentration was measured. Standard cholesterol solution $(300 \mathrm{mg} / \mathrm{dl}$, Asan Pharmaceutical, Korea) was serially diluted twofold from $300 \mathrm{mg} / \mathrm{dl}$ to $37.5 \mathrm{mg} / \mathrm{dl}$ with DI water and then $2 \mu l$ of each diluted solution was pipetted out in four tubes. $300 \mu l$ of COD-POD reagent with a mixing ratio of ChE:COD: $\mathrm{POD}=1: 1: 20$ were added to the four tubes and then they were stirred enough to be mixed. Thereafter, about $180 \mu l$ from each tube was transferred into the SRM chamber and kept in the dark at $37^{\circ} \mathrm{C}$ for $5 \mathrm{~min}$ before measuring the absorbance of LDL cholesterol. The measurement of the absorbance was done using the SRM system, and then its curve was plotted for ten successive times repeated experiments with the same preparation. As shown in Fig. 3, the experiment was conducted over a wide range of cholesterol levels of 30 to $300 \mathrm{mg} / \mathrm{dl}$, considering that the National Institute of Health recommends that a person's cholesterol level stays below $200 \mathrm{mg} / \mathrm{dl}$ and that the cholesterol levels 200-239

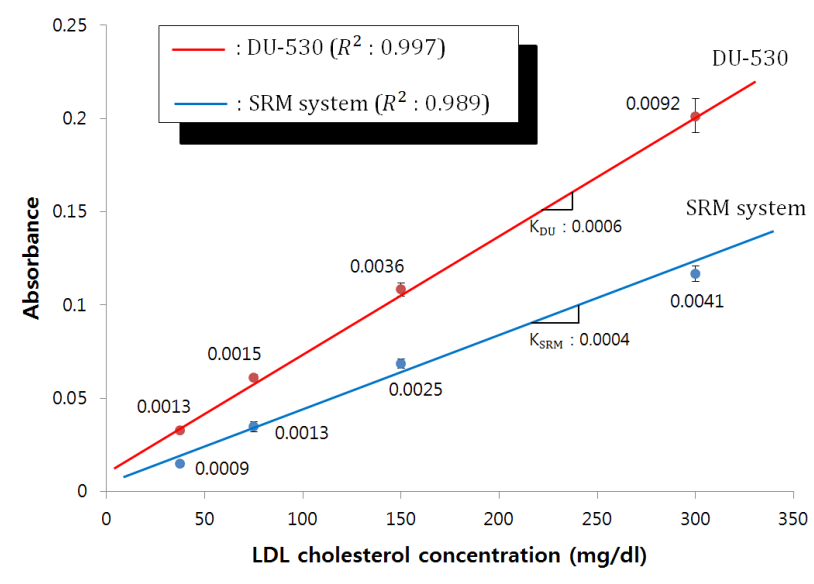

FIG. 3. Graphs of absorbance vs. LDL cholesterol concentration where the numbers on the curve denote the standard deviation of measurement at each concentration. The $\mathrm{K}_{D U}$ and $\mathrm{K}_{S R M}$ indicate the slope of the optical absorbance to LDL cholesterol concentration of DU-530 and SRM system, respectively.

$\mathrm{mg} / \mathrm{dl}$ are regarded to be borderline high. The curve shows a good linear relationship $\left(R^{2}=0.989\right)$ over a wide concentration range of 30 to $300 \mathrm{mg} / \mathrm{dl}$ with the average coefficient of variation $(\mathrm{CV}=4.2 \%)$, comparable to a commercialized spectrometer with the average $\mathrm{CV}$ equal to $3.6 \%$ (DU-530, Beckman, USA). In this figure, even though the SRM system seems to have a lower sensitivity to concentration when being compared to DU-530, eventually they have almost same performance in terms of both sensitivity and $\mathrm{CV}$ when the ratio (1.5) of $\mathrm{K}_{D U}$ to $\mathrm{K}_{S R M}$ is multiplied to the absorbance value of SRM. It is also noted that despite the fact that the path length of SRM is longer than that of DU-530, its performance is a little degraded because of the imperfect mirror reflection, but still giving the performance comparable to the DU-530.

\section{CONCLUSION}

We presented a novel detection device for determining the concentration of LDL cholesterol which uses a SRM buried in the detection chamber. Multiple beam reflections formed during the travel of the light through the chamber gave a path length long enough to determine the LDL cholesterol concentration with almost the same level of sensitivity as the spectrophotometer DU-530 (Beckman, USA). The experimental results show that our proposed SRM system is not only comparable to already commercialized spectrometers in terms of coefficient of variation and coefficients of determination $\left(R^{2}\right)$, but also able to be used as one of the promising approaches to spectrophotometrybased LOD that is as thin as a compact disc. In addition, even though the experiment was done only for LDL cholesterol, our SRM system could be easily adapted to any kinds of 
LOD. Future work will include a fully automated LOD for measuring cholesterol concentration in whole blood, and then reducing the crosstalk with other species in blood such as hemoglobin will be one of our primary works.

\section{REFERENCES}

1. J. R. Crouse, J. S. Parks, H. M. Schey, and F. R. Kahl, "Studies of low density lipoprotein molecular weight in human beings with coronary artery disease," Journal of Lipid Research 26, 566-574 (1985).

2. Z. Matharu, G. Sumana, M. K. Pandey, V. Gupta, and B. D. Malhotra, "Low density lipoprotein sensor based on surface plasmon resonance," Thin Solid Films 518, 719-723 (2009).

3. Z. Matharu, A. J. Bandodkar, G. Sumana, P. R. Solanki, E. M. I. M. Ekanayake, K. Kaneto, V. Gupta, and B. D. Malhotra, "Low density lipoprotein detection based on antibody immobilized self-assembled monolayer: Investigations of kinetic and thermodynamic properties," J. Phys. Chem. B 113, 14405-14412 (2009).

4. L. Charpentier and N. E. Murr, "Amperometric determination of cholesterol in serum with use of a renewable surface peroxidase electrode," Analytica Chimica Acta 318, 89-93 (1995).

5. T. Nakaminami, S. Ito, S. Kuwabata, and H. Yoneyama, "Amperometric determination of total cholesterol at gold electrodes covalently modified with cholesterol oxidase and cholesterol esterase with use of thionin as an electron mediator," Anal. Chem. 71, 1068-1076 (1999).

6. T. Nakaminami, S. Kuwabata, and H. Yoneyama, "Electrochemical oxidation of cholesterol catalyzed by cholesterol oxidase with use of an artificial electron mediator," Anal. Chem. 69, 2367-2372 (1997).

7. M. A. T. Gilmartin and J. P. Hart, "Fabrication and characterization of a screen-printed, disposable, amperometric cholesterol biosensor," Analyst 119, 2331-2336 (1994).

8. M. Situmorang, P. W. Alexander, and D. B. Hibbert, "Flow injection potentiometry for enzymatic assay of cholesterol woth a tungsten electrode sensor," Talanta 49, 639-679 (1999).

9. E. Koller, O. Quehenberger, G. Jurgens, O. S. Wolfbeis, and H. Esterbauser, "Investigation of human plasma low density lipoprotein by three-dimensional fluorescence spectroscopy," FEBS letters 198, 229-234 (1986).

10. K. Z. Liu, R. A. Shaw, A. Man, T. C. Dembinski, and H. H. Hantsch, "Reagent-free, simultaneous determination of serum cholesterol in HDL and LDL by infrared spectroscopy," Clinical Chemistry 48, 499-506 (2002).

11. B. S. Lee, Y. U. Lee, H. S. Kim, T. H. Kim, J. Park, J. G. Lee, J. T. Kim, H. S. Kim, W. G. Lee, and Y. K. Cho, "Fully integrated lab-on-a-disc for simultaneous analysis of biochemistry and immunoassay from whole blood," Lab Chip 11, 70-78 (2011).

12. D. C. Duffy, H. L. Gillis, J. Lin, N. F. Sheppard, Jr., and G. J. Kellogg, "Microfabricated centrifugal microfluidic systems: characterization and multiple enzymatic assays," Anal. Chem. 71, 4669-4678 (1999).

13. D. D. Nolte, "Invited review article: review of centrifugal microfluidic and bio-optical disks," Review of Scientific Instruments 80, 101101 (2009).

14. R. Corkin, M. Czugala, C. Rovira-Borras, J. Ducree, D. Diamond, and F. B. Lopez, "A wireless paired emitter detector diode device as an optical sensor for lab-on-a-disc applications," in Proc. Transducers'11 (Beijing, China, June 2011), pp. 2526-2529.

15. T. O. Tiffany, C. A. Burtls, J. C. Mailen, and L. H. Thacker, "Dynamic multicuvette fluorometer-spectrophotometer based on the GeMSAEC face analyzer principle," Anal. Chem. 45, 1716-1723 (1973).

16. J. Steigert, M. Grumann, T. Brenner, L. Riegger, J. Harter, R. Zengerle, and J. Ducree, "Fully integrated whole blood testing by real-time absorption measurement on a centrifugal platform," Lab chip 6, 1040-1044 (2006).

17. M. Grumann, I. Moser, J. steigert, L. Riegger, A. Geipel, C. Kohn, G. Urban, R. Zengele, and J. ducree, "Optical beam guidance in monolithic polymer chips for miniaturized colorimetric assays," in Proc. Micro Electro Mechanical Systems, MEMS 2005 (Miami, FL, USA, 2005), pp. 108-111. 